\title{
Switchable Helical Structures Formed by the Hierarchical Self-Assembly of Laterally Tethered Nanorods
}

Trung Dac Nguyen and Sharon C. Glotzer*

\author{
The formation of helical scrolls formed by self-assembly of tethered \\ nanorod amphiphiles and their molecular analogs are investigated. A model \\ bilayer sheet assembled by laterally tethered nanorods is simulated and \\ shown that it can fold into distinct helical morphologies under different \\ solvent conditions. The helices can reversibly transform from one \\ morphology to another by dynamically changing the solvent condition. This \\ model serves both to inspire the fabrication of laterally tethered nanorods \\ for assembling helices at nanometer scales and as a proof-of-concept for \\ engineering switchable nanomaterials via hierarchical self-assembly.
}

\author{
Keywords: \\ - helical structures \\ - hierarchical self-assembly \\ - switchable nanostructures \\ - tethered amphiphiles
}

\section{Introduction}

Fabrication of functional structures at nanometer and micrometer scales using bottom-up techniques such as directed- and self-assembly has gained increasing interest among the materials community in recent years. A popular approach to engineering a target structure involves designing building blocks such that their anisotropic interactions give rise to the desired microphase separation and local packing. To this end, shape molecules, ${ }^{[1]}$ block copolymers, ${ }^{[2]}$ amphiphilic peptides, ${ }^{[3]}$ and DNA- and polymer-functionalized nanoparticles $^{[4-7]}$ are popular building blocks in engineering ordered, functional nanostructures. However, it is challenging to control the self-assembly of many building blocks into higher-ordered structures from isotropic states because the system may easily become trapped in deep energy minima. A promising solution to help overcome this obstacle is to guide the assembly path by preparing intermediate preassembled patterns such that they can naturally fold into target structures. ${ }^{[8-10]}$ With this approach the final structures are no longer limited to classical surfactant-based morphologies but can be more diverse in shape and scale. For example, Ciszek et al. demonstrated that assemblies of amphiphilic gold-polypyrrole nanorods on intentionally designed two-

[*] Prof. S. C. Glotzer, T. D. Nguyen Department of Chemical Engineering and Department of Materials Science and Engineering University of Michigan

Ann Arbor, MI 48109 (USA)

E-mail: sglotzer@umich.edu

DOI: $10.1002 /$ smll.200900168 dimensional footprints, when released from the template, can form various desired higher-ordered structures including hemispheres, open spheres, and capsules under the same environmental conditions. ${ }^{[9]}$ Chen and co-workers showed that para-terphenylen-1,4"-ylenebis(dodecanamide) (TB) molecules consisting of a central rigid rod-like terphenylene segment, two secondary amido functional groups, and two flexible alkyl chains, self-assemble into nanosheets by $\pi-\pi$ stacking of terphenylene units and intermolecular hydrogen bonding between amido-functional groups. The sheets subsequently roll up to form nanotubes or stack into multi-layered sheets depending on the concentration of TB in tetrahydrofuran (THF). ${ }^{[10]}$ Recent computational and experimental studies have shown that laterally tethered nanorods in selective solvents ${ }^{[11]}$ and T-shaped rod-coil molecules in the melt ${ }^{[12]}$ selfassemble into bilayer sheets of $\mathrm{P}_{2}$ symmetry in which the rod segments are organized into crystalline, in-plane sublayers. The bilayer sheets subsequently scroll into spiral multilayered tubules with an outer diameter of $\sim 100 \mathrm{~nm}$ under melt conditions. $^{[12]}$

Helical structures are commonly found at molecular scales. Examples include natural biological molecules and synthetic polymers. However, little progress has been made to control the formation of helical structures at mesoscopic scales. It has been shown that helical structures spontaneously appear in chainlike molecules most likely because of the presence of directional hydrogen bonds at certain positions. ${ }^{[13-15]}$ More importantly, the formation of local hydrogen bonds, that is, between neighboring monomers, should be faster and more favorable than non-local bonds. ${ }^{[16]}$ Discrete building blocks can also form helices if their interactions are sufficiently anisotropic that a helical arrangement is the most energetically favored. ${ }^{[17,18]}$ 
Most attempts to date require decorating molecular building blocks with sticky or polar "patches" to induce directional noncovalent bonds ${ }^{[3,17]}$ or introducing lateral substituents at some specific positions on chain-like supramolecules to help guide the folding process ${ }^{[13,19]}$ The assembled helical structures are static in the sense that we cannot dynamically tune their geometrical parameters, for example, radius and pitch, once they are formed. For nanoparticle and colloidal systems, despite the increasingly expanded library of anisotropic building blocks, ${ }^{[20]}$ there have been few reported to selfassemble into helices. For instance, Yin et al. reported that spherical colloids can assemble into helices with pre-defined handedness in $\mathrm{V}$-shaped grooves by means of capillary forces. ${ }^{[21]}$ Price et al. proposed a method for fabricating metal helical nanostructures using a phospholipid microtubule template coated with oppositely charged polyelectrolytes in which the metal nanoparticles are electrostatically bound to the helical template through a negatively charged catalyst. ${ }^{[22]}$ Vanapalli and co-workers ${ }^{[23]}$ introduced a method for active assembly of spherical particles into a zigzag morphology - a two-dimensional helix - in confined rectilinear microchannels.

Herein we propose an alternative to assemble nanoscale helical structures from experimentally available building blocks, that is, laterally tethered nanorods and their analogs, without any confinement. We demonstrate the formation of three distinct helices that differ in pitch and radius from sheets assembled by laterally tethered nanorods depending on the solvent selectivity. Moreover, we show that the helices spontaneously transform from one stable helix to another when the solvent condition is dynamically changed. Specifically, when a solvent that is poor for rods is switched from good to poor for tethers and vice versa while maintaining solvent-rod immiscibility, the helices transform accordingly from one morphology to another. A similar phenomenon is observed when we change a solvent that is good for tethers from good to poor for rods while maintaining solvent-tether miscibility. When a solvent that is poor for tethers is switched from good to poor for rods, we also observe an expected change in the helix morphology, but the reverse transformation is prohibited, requiring an intermediate state.

\section{Model and Method}

\subsection{Model}

We use a minimal model of laterally tethered nanorods similar to the one used in our previous work. ${ }^{[11,12]}$ An example of a model bilayer sheet is shown in Figure $1 \mathrm{~A}$ and $\mathrm{B}$. The rods consist of three spherical beads with diameter $\sigma$ placed at a distance $\sigma$ apart. The center bead is linked with the coil via a finitely extensible non-linear elastic (FENE) spring. ${ }^{[24]}$ The tethers are modeled as linear chains of spherical beads with diameter $\sigma$, bonded via FENE springs. We combine the rods into rigid pairs and subsequently connect them with FENE springs to create a permanent and flexible bilayer. The rod pairs are arranged into a $\mathrm{P}_{2}$ symmetry packing with parallel sublayers as in References ${ }^{[11,12]}$ and the tethers extend out of the sheet surface. We use a bonding scheme, illustrated in Figure 1C, to maintain the sublayers during a simulation run. The bonds

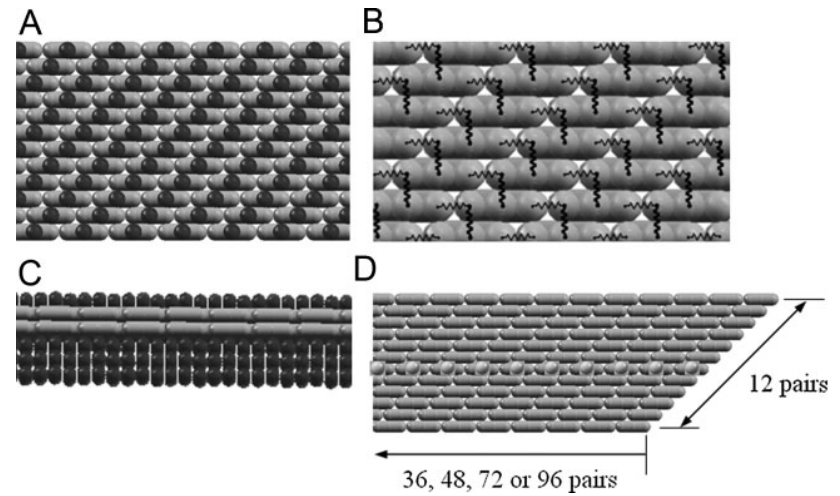

Figure 1. A portion of the model bilayer sheet used for the simulations. A) Side view. B) Top view: the rods in a pair (bright color) are rigidly grouped. Longer tethers consist of three beads. C) Bonding scheme: only the rods in the bottom layer in (B) are bonded. D) The backbone of the sheet used for calculating helical order parameter $\mathrm{H}_{4}$ consists of highlighted spheres. Bold springs represent $\pi-\pi$ interactions. Light springs represent end-to-end attractions. The dimensions of the sheet are measured in rod pairs. The tethers are removed for clarity in (C) and (D).

connecting rods in the same sublayers represent the side-to-side attraction between the rods within sublayers, and those connecting rod ends represent the end-to-end attraction between the rods from different sublayers. For laterally tethered nanorods and their molecular analogs, for example, T-shaped rod-coil molecules, the crystalline ordering of the rods within sublayers is favored over other arrangements because both the rod contacts and the free volume of the lateral tethers are maximized. Experiments have shown that once the sheets are formed, they are well maintained under scrolling. ${ }^{[12]}$ We hypothesize that the rod binding is much stronger than solvophilic forces, and hence can sustain the sheets under different solvent conditions. The bonds are therefore introduced to mimic the $\mathrm{P}_{2}$ symmetry packing of the rods and to retain the sheets during simulations while taking into account thermal fluctuations. This bonding scheme has been used to successfully reproduce the behavior of the bilayer sheets formed by $\mathrm{T}$-shaped rod-coil molecules ${ }^{[12]} \mathrm{We}$ therefore surmise that our model is valid as long as the local short-range attractions between the rod pairs are sufficiently strong to maintain the sublayers with a certain freedom of the rods within. To help guide the bending of the sheet toward one side, we choose different tether lengths on each surface of the sheet: the longer tethers on one surface consist of three beads, and the shorter tethers on the other surface consist of one bead. It is important to note that we only bond the rods on the surface with longer tethers; otherwise the sheet cannot bend because of the balance in the stiffness of its two surfaces. This is realistic despite excluded volume arguments because the packing frustration of the longer tethers is reduced by the rod tilt within the $\mathrm{P}_{2}$ packing of the sublayers. Under each solvent condition, we also run simulations with different lengths of the longer tethers to investigate the dependence of helix geometry on tether bulkiness.

The adjustable environmental conditions include various parameters such as temperature, pressure, ionic strength, or $\mathrm{pH}$ of the solution and solvent selectivity. Previous simulations have investigated the effects of thermodynamic parameters, 
for example, temperature and pressure, on the phase behavior of tethered nanoparticle systems at different concentrations. ${ }^{[11,25-28]}$ In this study, we focus on solvent selectivity because it can be readily controlled by experiments and, more importantly, generalized to other external effects in terms of effective interactions between the species in the system. To take into account the solvent selectivity, we utilize empirical potentials that are successful in capturing the relevant physics of block copolymers, surfactants, and colloidal particles. ${ }^{[29,30]}$ If the solvent is poor for a species aggregation of that species will occur. To model the aggregation, the interaction between the beads of that species is modeled by the 12-6 Lennard-Jones potential truncated and shifted to zero at the cut-off distance of $2.5 \sigma$ to incorporate the short-range attraction and excluded volume interactions. If the solvent is good for a species, aggregation will not occur, and thus the interaction between the beads of that species is modeled by the softly repulsive WeeksChandler-Andersen (WCA) potential capturing only shortrange repulsion and excluded volume. The interaction between the rods and the tethers is always modeled by the WCA potential to represent their immiscibility. In our simulations, the potential-energy-well depths are chosen to be identical for rod-rod, tether-tether, and rod-tether interactions $\varepsilon_{\mathrm{R}-\mathrm{R}}=\varepsilon_{\mathrm{T}-\mathrm{T}}=\varepsilon_{\mathrm{R}-\mathrm{T}}=\varepsilon$. We investigate three solvent selectivity conditions for which the effective interactions between rods and tethers are listed in Table 1 . The natural units for these systems are the bead diameter, $\sigma$, the bead mass, $m$, and the Lennard-Jones well depth, $\varepsilon$. The timescale is accordingly defined as $\tau=\sigma(m / \varepsilon)^{1 / 2}$.

\subsection{Method}

We use Brownian dynamics (BD) to simulate the model bilayer sheets in three dimensions. In BD, solvent molecules are not explicitly included but instead implicitly represented by their effects on the equation motion of each bead. Details of this method can be found in References ${ }^{[26,27]}$. We choose the friction coefficient $\gamma=1.0$ to limit the ballistic motion of a bead in a time step to approximately $1.0 \sigma$. The rotational degrees of freedom of the rod pairs are incorporated using the equations for rotation of rigid bodies with quaternions. We employ the velocity Verlet scheme to integrate the equation of motion of the tether beads and to advance the rotational motion of the rod pairs with a time step $\Delta t=0.002 \tau$.

We investigate the model bilayer sheets with aspect ratios 36:12, 48:12, 72:12, and 96:12, measured in rod pairs. The sheets are initialized at their most stretched configuration in a large simulation box of which all the dimensions exceed the length of the sheets, and relaxed for a sufficient number of time steps

Table 1. Solvent selectivity and effective interactions between rods and tethers.

\begin{tabular}{cccccc}
\hline Case & \multicolumn{2}{c}{$\begin{array}{c}\text { Solvent } \\
\text { selectivity }\end{array}$} & \multicolumn{3}{c}{ Effective interactions } \\
\hline & Rod & Tether & Rod-Rod & Rod-Tether & Tether-Tether \\
\hline A & poor & good & attractive & non-attractive & non-attractive \\
B & poor & poor & attractive & non-attractive & attractive \\
C & good & poor & non-attractive & non-attractive & attractive \\
\hline
\end{tabular}

$(\approx 5000000)$ under athermal conditions for which the interactions between all species are non-attractive. The sheets are subsequently equilibrated with the chosen interactions at constant temperature $T=1.0 \varepsilon / k_{\mathrm{B}}$. For each solvent condition and sheet aspect ratio, we run several independent simulations (with different random number seeds) to ascertain that the final structures are reproducible. Final structures are determined by observing fluctuations in the system potential energy of less than $5 \%$ and a morphology that does not substantially change over a timescale of $\approx 50$ million time steps, which exceeds the relaxation time at equilibrium. Our simulations are conducted using LAMMPS, an open-source parallel molecular dynamics code. ${ }^{[31]}$ It takes approximately 8 days to complete 60 million time steps for a 96:12 sheet on two dual-core Apple G5 (2.0 GHz, 2 GB RAM) myrinet-connected nodes. All the results presented are for sheets with longer tethers consisting of three beads unless otherwise indicated.

\section{Results}

We observe the spontaneous formation of helical structures in all solvent selectivity conditions studied. For a given set of interactions, the bilayer sheets fold into helices with the same chirality, pitch, and radius for all investigated aspect ratios. Table 2 gives a summary of the geometry of the helices formed in our simulations. The small standard deviations in the pitch and radius relative to the average values indicate well-defined helical structures in all cases. To characterize the helical structures, we use an order parameter that is commonly used to represent the net helical growth in chainlike molecules, ${ }^{[32]}$ as defined by

$\mathrm{H}_{4}=\left(\frac{1}{\mathrm{~N}-2} \sum_{\mathrm{i}=2}^{\mathrm{N}-1} \mathbf{u}_{i}\right)^{2}$

where $\mathbf{u}_{i}$ is the unit vector proportional to $\left(\mathbf{r}_{i}-\mathbf{r}_{i-1}\right) \times\left(\mathbf{r}_{i+1}-\mathbf{r}_{i}\right), \mathbf{r}_{i}$ is the position of the $i^{\text {th }}$ monomer and $N$ is the length of the sheet, measured in rod pairs. Here we define the "monomers" as the center bead of rods that lies in the center row parallel to the longer edge of the sheet (Figure 1D). The time evolution of $\mathrm{H}_{4}$ in different simulation runs illustrated in Figure 2 shows the dynamic behavior of helix formation, indicating a noticeably sharp transition to helical states in Case B and Case C. The small absolute value of

Table 2. Geometric parameters of the helices formed in three solvent conditions. The pitches and radii are averaged over 10 measurements along the backbone of the sheets and aspect ratios. The errors are the standard deviation from the average values.

\begin{tabular}{|c|c|c|c|}
\hline Case & Effective interactions & Pitch $[\sigma]$ & Radius $[\sigma]$ \\
\hline A & $\begin{array}{l}\text { Rod-rod attractive } \\
\text { Tether-tether non-attractive }\end{array}$ & $77.9 \pm 0.91$ & $10.2 \pm 1.48$ \\
\hline$B$ & $\begin{array}{l}\text { Rod-rod attractive } \\
\text { Tether-tether attractive }\end{array}$ & $34.7 \pm 0.82$ & $10.4 \pm 0.73$ \\
\hline C & $\begin{array}{l}\text { Rod-rod non-attractive } \\
\text { Tether-tether attractive }\end{array}$ & $20.3 \pm 0.51$ & $3.8 \pm 0.65$ \\
\hline
\end{tabular}




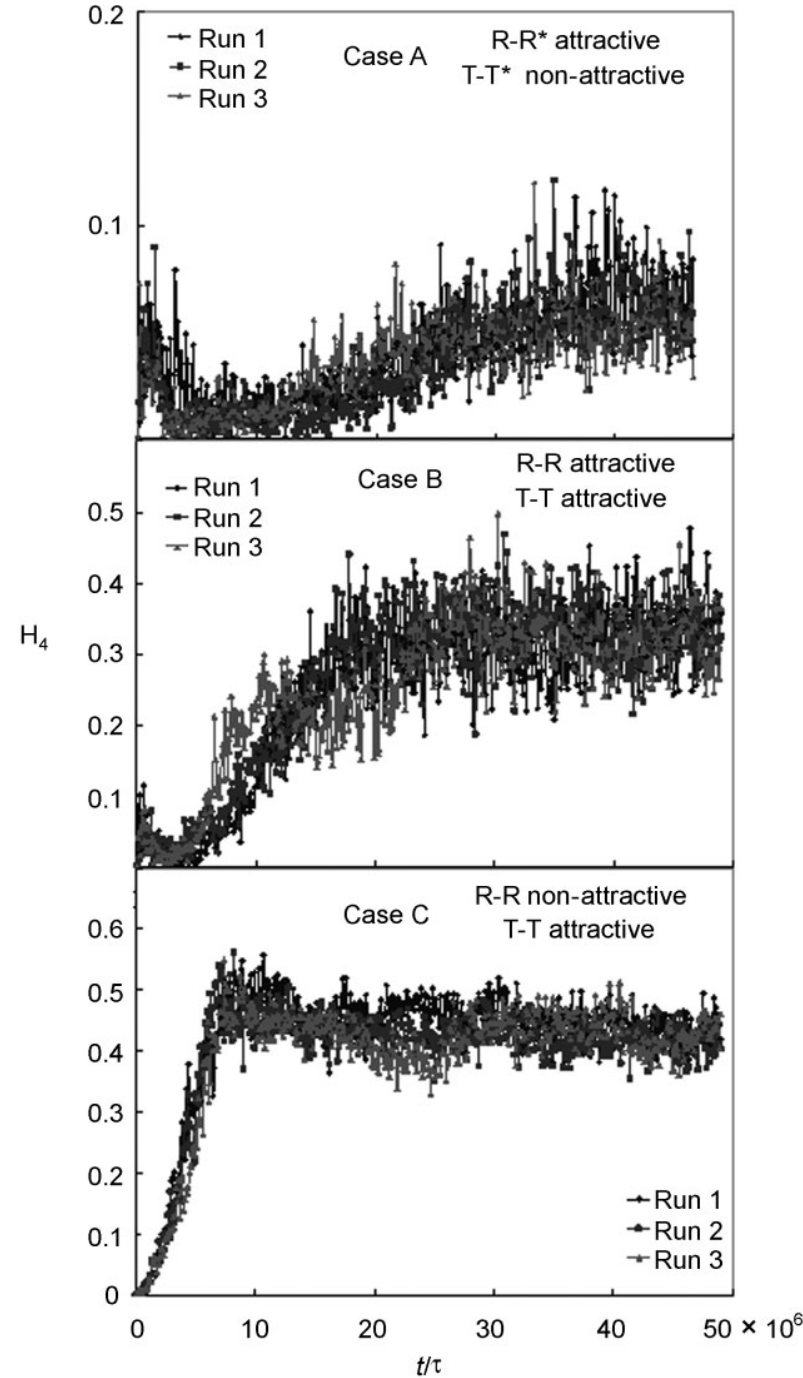

Figure 2. Representative time evolution of the helical order parameter $\mathrm{H}_{4}$ of the backbone of a 72:12 bilayer sheet in three different runs in three solvent conditions. R: rod; T: tether.

$\mathrm{H}_{4}$ in the helical state for Case A is due to the large pitch as compared to the radius of the helix, as illustrated by the backbone shape in Figure 4. As shown in Figure 3, a typical helix forms spontaneously from curling at the two ends of the sheet. Only one handedness is observed, as imposed by the rod orientation in sublayers. Defects may occur if the sheet length does not match the number of helical turns. Simulation snapshots (Figure 4) together with the average pitch and radius

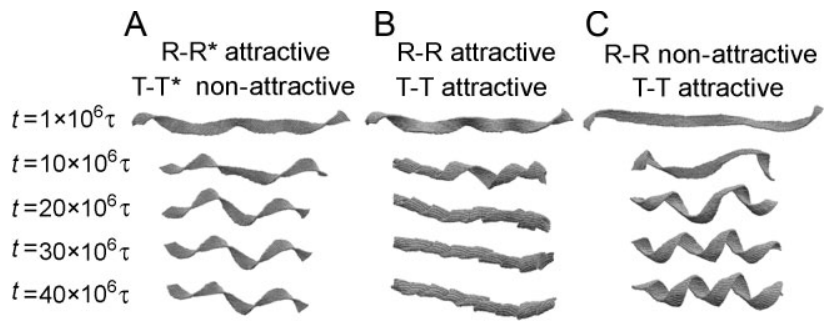

Figure 3. An example time evolution of a $96: 12$ bilayer sheet in three solvent conditions. The tethers are not shown for clarity. R: rod; T: tether

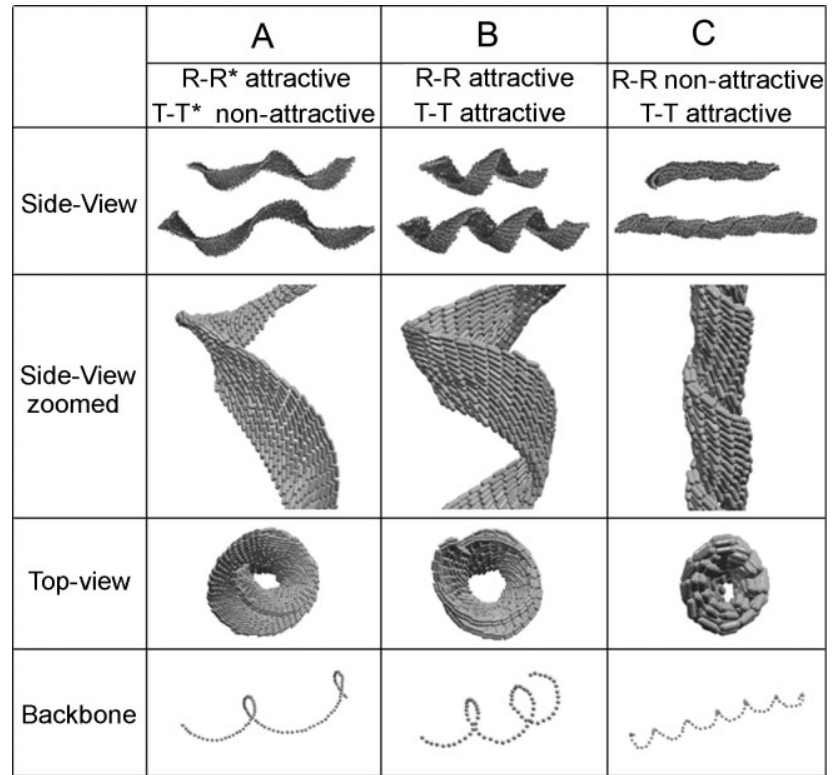

Figure 4. Helical structures formed under three solvent conditions, as indicated in Table 2. A) Case A, B) Case B, C) Case C. In the zoomed-in sideview and top-view image, the tethers are not shown for clarity. R: rod, $\mathrm{T}$ : tether.

(Table 2) reveal that the helical morphology substantially varies with the solvent selectivity.

\subsection{Case A: Solvent Good for Tethers and Poor for Rods}

When the solvent is good for the tethers and poor for the rods, the rods are attractive to each other while the tethers are not. Previous simulations showed that laterally tethered nanorods assemble into bilayer sheets under these conditions. ${ }^{[11]}$ When released into a large volume, the sheets spontaneously scroll into helical structures $\left(\mathrm{H}_{\mathrm{A}}\right)$, as depicted in Figure 4. To maximize the free volume of the long tethers on one surface, the sheet bends toward the opposite surface with shorter tethers. Meanwhile, the rod pairs reorient within the sublayers to maximize their contacts and reduce the tether grafting density on both sides of the sheet. The rod pairs close to the center of the helix are nearly parallel to the scrolling axis, aligning with the rod pairs from adjacent sublayers. The sheet forms an open helix with a large pitch, as shown in the backbone image in Figure 4. These results suggest that we can tune the helix geometry by changing the entropic effects of the tethers through their bulkiness or grafting density. For example, additional simulations demonstrate that if we increase the length of the longer tethers to four, five, or six beads, the pitch will increase accordingly while the radius does not change significantly (Table 3 ).

\subsection{Case B: Solvent Poor for Tethers and Rods}

When the solvent is poor for both species, rods attract rods and tethers attract tethers. The interactions between the rods and tethers in this case resemble those in a melt, which result in $\mathrm{P}_{2}$ symmetry packing of rods within bilayer sheets. ${ }^{[12]}$ In contrast with Case $\mathrm{A}$, the net attraction between the long tethers is larger than that between the shorter ones, favoring 
Table 3. Geometric parameters of the helices formed in three solvent conditions with different, longer tether lengths. The pitches and radii are averaged over 10 measurements along the backbone of the sheets and aspect ratios. The errors are the standard deviation from the average values.

\begin{tabular}{|c|c|c|c|c|c|c|}
\hline \multirow[b]{3}{*}{ Tether length, $\sigma$} & \multicolumn{2}{|c|}{ Case A } & \multicolumn{2}{|c|}{ Case B } & \multicolumn{2}{|c|}{ Case C } \\
\hline & \multicolumn{2}{|c|}{$\begin{array}{l}\text { Rod-rod attractive } \\
\text { Tether-tether non-attractive }\end{array}$} & \multicolumn{2}{|c|}{$\begin{array}{l}\text { Rod-rod attractive } \\
\text { Tether-tether attractive }\end{array}$} & \multicolumn{2}{|c|}{$\begin{array}{l}\text { Rod-rod non-attractive } \\
\text { Tether-tether attractive }\end{array}$} \\
\hline & Pitch $[\sigma]$ & Radius $[\sigma]$ & Pitch $[\sigma]$ & Radius $[\sigma]$ & Pitch $[\sigma]$ & Radius $[\sigma]$ \\
\hline 4 & $84.4 \pm 0.42$ & $13.3 \pm 0.28$ & $36.6 \pm 0.89$ & $10.6 \pm 0.84$ & $19.8 \pm 0.94$ & $4.8 \pm 0.48$ \\
\hline 5 & $91.3 \pm 0.26$ & $12.5 \pm 0.78$ & $30.6 \pm 0.39$ & $10.6 \pm 0.33$ & $19.1 \pm 0.47$ & $5.2 \pm 0.39$ \\
\hline 6 & $94.8 \pm 0.58$ & $12.3 \pm 0.83$ & $26.7 \pm 0.55$ & $9.9 \pm 0.49$ & $19.8 \pm 0.41$ & $5.5 \pm 0.22$ \\
\hline
\end{tabular}

the bending of the sheet towards the long tether side. Since bending along the rod direction is highly restricted by the increase in the grafting density of the tethers, the sheet tends to fold along the sublayer directions instead. The sheet therefore curves into a helical structure $\left(\mathrm{H}_{\mathrm{B}}\right)$ with a smaller pitch as compared to $\mathrm{H}_{\mathrm{A}}$. Moreover, the attraction between the long tethers and the immiscibility between rods and tethers result in the short tethers on the outer surface, consistent with a geometric packing factor analysis. ${ }^{[33]}$ The side-view image further shows that the sheet slightly twists to reduce the grafting density of the long tethers. Though the tethers are attractive their aggregation is limited by the strong attraction between the rod pairs, which comes collectively from both sides of the sheet, and by the excluded volume of the long tethers. As a result, the sublayers cannot fold tightly on each other but instead must adopt a curvature that accommodates these competing forces. Additional simulations show that the radius of the helices is almost invariant as the length of the longer tethers is increased. This indicates that the bending of the sheet is strongly influenced by the net attraction of the rod pairs within the sheet. As the tether length increases, the attraction between longer tethers becomes stronger, pulling the helical turns closer together, and hence reducing the helix pitch (Table 3 ).

\subsection{Case C: Solvent Poor for Tethers and Good for Rods}

When the solvent is poor for the tethers and good for the rods, the rods are non-attractive while the tethers are attractive. It should be clarified that this solvent selectivity does not support the self-assembly of sheets from precursor tethered nanorod building blocks, and thus may be considered only after the sheet is already formed, for example, under either of the solvent conditions described above. Under these conditions, the sheet then folds tightly into a helical structure $\left(\mathrm{H}_{\mathrm{C}}\right)$, as shown in Figure 4. Similar to Case B, the attraction between the long tethers leads to the folding of the sheet along the sublayer separations. However, since the rod pairs are non-attractive the sheet can bend more tightly as compared to Case B, until densely packed in the interior. Additional simulations reveal that the helical pitch is relatively independent of the tether length and approximates the width of the sheet, whereas the interior radius can be tuned through varying the long tether length (Table 3).

\section{Discussion}

Of particular importance is the driving force for the spontaneous formation of helical structures in our bilayer sheet model at the initial stage. Sabeur et al. demonstrated that a stretched hydrophobic chain easily falls into a helical state as a local energy minimum if hydrodynamic effects are sufficiently screened. ${ }^{[34]}$ In our simulations, these necessary conditions are fulfilled. First, since the model bilayer sheet is somewhat analogous to a linear but "flat" homopolymer where the sublayers are sequentially connected via springs it is reasonable that the instability of the initial stretched configuration of the sheet triggers the helix formation. Second, the difference in the tether lengths on the two surfaces drives the sheet to bend and fold toward one side, mimicking the hydrophobic effects in chainlike molecules. Third, hydrodynamics is certainly suppressed in our BD simulation method due to the uncorrelated random forces acting on the beads. Since the helices are reproducibly formed at a much higher temperature $\left(T=1.0 \varepsilon / k_{\mathrm{B}}\right)$ as compared to homopolymers, ${ }^{[34]}$ we argue that the latter condition can be relaxed because at that temperature the system is mainly diffusive, and thus the role of hydrodynamic interactions becomes negligible. It follows that our simulations can reasonably describe the dynamics of the system without hydrodynamic interactions. The ability of helices to form at such a high temperature may be due to the crystalline packing of rods within sublayers, which help direct the chirality of the sheet, as compared to the isotropic nature of homopolymers.

Because the helical states in all solvent conditions studied are robust under the bombardment of implicit solvent molecules, it is reasonable to state that they are separated from other local energy minima by barriers at least greater than the thermal energy, $k_{\mathrm{B}} T$. Intriguingly, we observe that it is possible to switch between these helical states by varying the solvent selectivity. Starting with a helical structure already formed under a given condition, we change the interaction potentials between species to represent the target solvent condition. Our simulations reveal that the helices $\mathrm{H}_{\mathrm{A}}$ and $\mathrm{H}_{\mathrm{B}}$ can easily transform into each other, as can $\mathrm{H}_{\mathrm{A}}$ and $\mathrm{H}_{\mathrm{C}}$. The only exception is that while $\mathrm{H}_{\mathrm{B}}$ can be changed into $\mathrm{H}_{\mathrm{C}}$, the reverse direction is impossible, at least computationally. The reversible transition from $\mathrm{H}_{\mathrm{A}}$ to $\mathrm{H}_{\mathrm{B}}$ and $\mathrm{H}_{\mathrm{C}}$ results from the fact that, unlike in the latter cases, the non-attractive to tethers in $\mathrm{H}_{\mathrm{A}}$ tend to segregate to maximize their free volume. When the interactions between the tethers are switched from non-attractive to attractive or vice versa, the sheet easily tevolves without being trapped in the intial configuration. In Case B and Case C, however, the tethers are always attractive and tend to aggregate to maximize their contacts. Consequently, when the structure is initialized in $\mathrm{H}_{\mathrm{C}}$, it immediately becomes trapped in this state even when the rods turn from non-attractive to attractive. We also notice the 
negligible difference in the system potential energy between $\mathrm{H}_{B}$ and $\mathrm{H}_{\mathrm{C}}$ in the solvent condition of Case $\mathrm{B}$, that is, less than $0.5 \varepsilon$ per bead. A direct transition from $\mathrm{H}_{\mathrm{C}}$ to $\mathrm{H}_{\mathrm{B}}$ is therefore entropically prohibitive and cannot occur in our simulations. However, the transition from $\mathrm{H}_{\mathrm{C}}$ to $\mathrm{H}_{\mathrm{B}}$ might be carried out indirectly through $\mathrm{H}_{\mathrm{A}}$, that is, transforming $\mathrm{H}_{\mathrm{C}}$ into $\mathrm{H}_{\mathrm{A}}$, and subsequently to $\mathrm{H}_{\mathrm{B}}$ by varying the solvent selectivity appropriately. This is an example of designing a reversible pathway to switch from one structure to another by judiciously adjusting external conditions.

The folding process of the bilayer sheet into helical structures is essentially kinetically driven, similar to that of pre-assembled two-dimensional patterns into hemispheres and open spheres. ${ }^{\left[{ }^{9]}\right.}$ Consequently, the initial stretched configuration of the bilayer sheets is critical to the formation of the helices. Despite the isotropic interactions in our minimal model, the chirality of the final structures arises due to the $\mathrm{P}_{2}$ symmetry packing of the rod pairs, which helps to guide the folding direction of the sheet. However, the anisotropic effect of the liquid-crystalline sublayers on the folding direction can be diminished if the number of the rod pairs in each sublayer is smaller than a certain value, and the sheet behaves as a chainlike molecule. For example, additional simulations indicate that 48:6 and 48:8 bilayer sheets cannot form regular helices but instead form a wormlike morphology with locally helical segments in Case A and Case B. Other factors that require further elaborate investigations outside the scope of this paper involve the rod aspect ratio and relative strengths of interactions between rods and tethers. These parameters are likely to play important roles in controlling the curvature, pitch, and radius of the helices. Our results also suggest that a simplified two-state model may be constructed to describe the transition from extended bilayer (described as a semiflexible membrane) to the scrolled state. In such a model, the $\mathrm{P}_{2}$ packing of the rods that controls the handedness and pitch could be included in a continuum description via an anisotropic elasticity term.

\section{Conclusions}

We have proposed a model bilayer sheet formed by laterally tethered nanorods and their analogs that reproducibly forms regular helical structures with a unique handedness. Depending on the solvent selectivity, distinct helical morphologies spontaneously emerge. When the solvent is good for the tethers and poor for the rods, the sheet twists into a helix with a large pitch. When the solvent is poor for both species, the sheet folds along the sublayer separations, resulting in a helix with a smaller pitch and larger interior radius. When the solvent is poor for the tethers and good for the rods, the sheet forms a helix with the interior filled by the tethers. Under a given solvent condition, we demonstrate that the helical structure can be further tuned by adjusting the tether length on one side of the sheet. This work can be used to guide the synthesis of helical structures at nano- and microscales from pre-formed sheetlike assemblies. We further show that a helical morphology can be spontaneously switched from one structure to another when the solvent condition is dynamically changed. The model bilayer sheet can serve therefore as a proof-of-concept for fabricating switchable nanomaterials.

\section{Acknowledgements}

We thank C. R. lacovella and A. Santos for helpful comments on the manuscript, and acknowledge financial support by the Air Force Office of Scientific Research under MURI grant FA955006-1-0337. T.D.N also acknowledges the Vietnam Education Foundation.

[1] R. A. Reddy, C. Tschierske, J. Mat. Chem. 2006, 16, 907.

[2] F. S. Bates, G. H. Fredrickson, An. Rev. Physi. Chem. 1990, 41, 525.

[3] S. Zhang, D. M. Marini, W. Hwang, S. Santoso, Current Opin. Chem. Biol. 2002, 6, 865 .

[4] A. P. Alivisatos, K. P. Johnsson, X. Peng, T. E. Wilson, C. J. Loweth, M. P. Bruchez, Jr, P. G. Schultz, Nature 1996, 382, 609.

[5] S. Park, J.-H. Lim, S.-W. Chung, C. A. Mirkin, Science 2004, 303, 348

[6] Z. Nie, D. Fava, E. Kumacheva, S. Zou, G. C. Walker, M. Rubinstein, Nat. Mater. 2007, 6, 609.

[7] J.-H. Ryu, M. Lee, Struc. Bond. 2008, 128, 63.

[8] D. H. Gracias, V. Kavthekar, J. C. Love, K. E. Paul, G. M. Whitesides, Adv. Mater. 2002, 15, 235.

[9] J. W. Ciszek, L. Huang, Y. Wang, C. A. Mirkin, Small 2008, 4, 206.

[10] Y. Chen, B. Zhu, F. Zhang, Y. Han, Z. Bo, Angew. Chem. Int. Ed. 2008, 47, 6015.

[11] M. A. Horsch, Z. Zhang, S. C. Glotzer, Nano Lett. 2006, 6, 2406.

[12] D.-J. Hong, E. Lee, H. Jeong, J. Lee, W.-C. Zin, T. D. Nguyen, S. C. Glotzer, M. Lee, Angew. Chem. Int. Ed. 2009, 48, 1664.

[13] J. C. Nelson, J. G. Saven, J. S. Moore, P. G. Wolynes, Science 1997, $277,1793$.

[14] J. Borg, M. H. Jensen, K. Sneppen, G. Tiana, Phys. Rev. Lett. 2001, 86,1031

[15] J. P. Kemp, Z. Y. Chen, Phys. Rev. Lett. 1998, 81, 3881.

[16] R. A. Bertsch, N. Vaidehi, S. I. Chan, W. A. Goddard, III, Proteins: Struct. Funct. Gen. 1998, 33, 343.

[17] H. M. Keizer, R. P. Sijbesma, Chem. Soc. Rev. 2005, 34, 226.

[18] S. N. Fejer, D. J. Wales, Phys. Rev. Lett. 2007, 99, 086106.

[19] H.-J. Kim, E. Lee, M. G. Kim, M.-C. Kim, M. Lee, E. Sim, Chem. Eur. J. 2008, 14, 3883

[20] S. C. Glotzer, M. J. Solomon, Nat. Mater. 2007, 6, 557.

[21] Y. Yin, Y. Xia, J. Am. Chem. Soc. 2003, 125, 2048.

[22] R. R. Price, W. J. Dressick, A. Singh, J. Am. Chem. Soc. 2003, 125, 11259.

[23] S. A. Vanapalli, C. R. Iacovella, K. E. Sung, D. Mukhija, J. M. Millunchick, M. A. Burns, S. C. Glotzer, M. J. Solomon, Langmuir 2008, 24, 3661.

[24] G. S. Grest, K. Kremer, Phys. Rev. A 1986, 33, 3628.

[25] Z. Zhang, M. A. Horsch, M. H. Lamm, S. C. Glotzer, Nano Lett. 2003, 3, 1341 .

[26] C. R. lacovella, M. A. Horsch, Z. Zhang, S. C. Glotzer, Langmuir 2005, 21, 9488

[27] M. A. Horsch, Z. Zhang, S. C. Glotzer, Phys. Rev. Lett. 2005, 95, 056105.

[28] a) T. D. Nguyen, Z. Zhang, S. C. Glotzer, J. Chem. Phys. 2008, 129, 244903; b) E. R. Chan, X. Zhang, C. Y. Lee, M. Neurock, S. C. Glotzer, Macromolecules 2005, 38, 6168; c) X. Zhang, E. R. Chan, S. C. Glotzer, J. Chem. Phys. 2005, 123, 184718; d) E. R. Chan, L. C. 
Ho, S. C. Glotzer, J. Chem. Phys. 2006, 125, 064905; e) X. Zhang, Z.-L. Zhang, S. C. Glotzer, Nanotechnology 2007, 18, 115706; f) C. R. Iacovella, S. C. Glotzer, Nano Lett. 2009, 9, 1206.

[29] T. Soddemann, B. Dunweg, K. Kremer, Eur. Phys. J. E 2002, 6, 409.

[30] S. C. Glotzer, M. Solomon, N. Kotov, AlChE J. 2004, 50, 2978.

[31] S. J. Plimpton, J. Comp. Phys. 1995, 117, 1.

[32] J. P. Kemp, Z. Y. Chen, Biomacromolecules 2001, 2, 389.
[33] J. N. Israelachvili, Intermolecular and Surface Forces, Academic, London 1992.

[34] S. A. Sabeur, F. Hamdache, F. Schmid, Phys. Rev. E 2008, 77, 020802.

Received: January 20, 2009

Revised: March 27, 2009

Published online: May 28, 2009 